\title{
Note sur la sociologie religieuse dans l'Année Sociologique
}

\section{Gabriel Le Bras}

\section{(2) OpenEdition \\ Journals}

Édition électronique

URL : http://journals.openedition.org/assr/23025

ISSN : $1777-5825$

\section{Éditeur}

Éditions de l'EHESS

Référence électronique

Gabriel Le Bras, "Note sur la sociologie religieuse dans l'Année Sociologique », Archives de sciences sociales des religions [En ligne], L'héritage des Formes, mis en ligne le 29 novembre 2013, consulté le 19 avril 2019. URL : http://journals.openedition.org/assr/23025 


\section{Note sur la sociologie religieuse dans l'Année Sociologique}

In: Archives des sciences sociales des religions. N. 21, 1966. pp. 47-53.

Citer ce document / Cite this document :

Le Bras Gabriel. Note sur la sociologie religieuse dans I'Année Sociologique. In: Archives des sciences sociales des religions. N. 21, 1966. pp. 47-53.

doi : 10.3406/assr.1966.2584

http://www.persee.fr/web/revues/home/prescript/article/assr_0003-9659_1966_num_21_1_2584 


\section{NOTE SUR LA SOCIOLOGIE RELIGIEUSE DANS L'ANNÉE SOCIOLOGIQUE}

GondéE en 1896 par Emile Durkheim, L'Année sociologique a paru en trois
séries, que séparent de longs intervalles de temps.

De 1896 à 1906, 10 volumes annuels se sont succédés au rythme d'abord prévu. Ils contiennent des Mémoires originaux et des Analyses d'ouvrages. Pour économiser le temps des collaborateurs, Durkheim décida en 1906 que la revue serait triennale et devrait se borner aux Analyses (1): les volumes XI (1906-1909) et XII (1909-1912) répondent à ce plan.

La guerre interrompit la publication, qui ne fut reprise qu'en 1923. Pendant ces dix ans, l'équipe avait été décimée; son chef mourut le 15 novembre 1917. Deux volumes seulement forment la seconde série (2).

La nouvelle interruption dura 23 ans. De 1948 à 1963, 12 volumes ont été publiés. Le dessein du Conseil d'administration est de rétablir autant que possible l'annualité - et la régularité, que diverses circonstances ont à certains moments, empêchée.

Durkheim entendait faire place à toutes les branches de la sociologie. Une douzaine de Mémoires (sur 25) concernent uniquement ou partiellement la sociologie religieuse. Quant aux Analyses, une section très copieuse et dont les divisions ont varié (3) lui est consacrée. Cette section a été l'œuvre de Marcel

(1) A.S., XI, préface.

(2) Au volume I (1923-1924), 9 nécrologies et M. Mauss, "L'œuvre inédite de Durkheim et de ses collaborateurs ", pp. 7-29.

(3) Hubert et Mauss se sont expliqué dans A.S., V, pp. 189-191, sur ces variations. Ils pensent qu'une classification idéale devrait répartir l'ensemble des phénomènes religieux sous quatre rubriques : représentations, pratiques, organisation, systèmes. En fait les $t$. I et XII sont ainsi divisés :

Tome I : I. Traités généraux, philosophie, méthode; II. Religions primitives en général; III. Culte domestique; IV. Croyances et pratiques concernant les morts; V. Cultes populaires en général, plus particulièrement agraires ; VI. Le rituel ; VII. Mythes ; VIII. Organisation du culte, monachisme; Notices diverses sur les grandes religions en général.

Tome XII : I. Traités généraux. Philosophie religieuse; II. Systèmes religieux des sociétés inférieures ; III. Systèmes religieux nationaux ; IV. Systèmes religieux universalistes; V. Systèmes religieux des groupes secondaires; VI. Cultes spéciaux; VII. Croyances et pratiques dites populaires; VIII. Croyances et rites concernant les morts ; IX. Magie; X. Rituel ; XI. Objets et lieux de culte; XII. Représentations religieuses; XIII. Les sociétés religieuses, leur droit et leur morale. 


\section{ARCHIVES DE SOCIOLOGIE DES RELIGIONS}

Mauss, avec la collaboration d'H. Hubert ( 3 bis) : il va de soi que nous retiendrons, parmi plusieurs centaines, les seules analyses qui contiennent une discussion originale.

Nous arrêterons notre relevé à 1940, c'est-à-dire à la fin de la seconde série, pour n'avoir qu'exceptionnellement à mentionner des hommes encore vivants, et parce que notre intention est seulement de reconnaître la part de l'Année Sociologique dans la naissance et le développement d'une véritable Ecole française de Sociologie. La contribution des deux grands animateurs dont l'œuvre a été ou sera le sujet d'articles particuliers (4), nous nous proposons de la situer simplement dans la Revue et nous évoquerons ceux qui les ont secondés.

Pour rendre compte de ce vaste labeur, aucune division, même celles des Analyses ne nous a paru satisfaisante (5). Nous croyons profitable de rassembler les réflexions de nos auteurs sous quatre chefs : domaine, méthode et sources de la sociologie des religions ; religions primitives, ethniques et universelles ; mythes, rites et systèmes; organisations des rapports intérieurs, extérieurs et supérieurs $(6)$.

Dans le second volume, Emile Durkheim traitait $D e$ la définition des phénomènes religieux (7): "croyances obligatoires" et "pratiques relatives aux objets donnés dans ces croyances", "subsidiairement croyances et pratiques facultatives qui concernent des objets similaires ou assimilés aux précédents ». Ni l'idée de Dieu, ni le surnaturel ne seraient de l'essence de la religion.

Telle est l'ampleur et la puissance de la religion qu'elle est à l'origine de toutes les activités collectives. "La religion contient en elle dès le principe, mais à l'état confus, tous les éléments qui, en se dissociant, en se déterminant, en se combinant de mille manières avec eux-mêmes, ont donné naissance aux diverses manifestations de la vie collective. C'est des mythes et légendes que sont sorties la science et la poésie, c'est de l'ornemantique religieuse et des cérémonies du culte que sont venus les arts plastiques. Lc droit et la morale sont nés des pratiques rituelles. On ne peut comprendre notre représentation de monde, nos conceptions philosophiques sur l'âme, sur l'immortalité, sur la vie, si l'on ne connaît les croyances religieuses qui en ont été la forme première. La parenté a commencé par être un lien essentiellement religieux; la peine, le contrat, le don, l'hommage sont des transformations du sacrifice expiatoire, contractuel, communiel, honoraire, etc... Tout au plus peut-on se demander si l'organisation économique fait exception et dérive d'une autre source; quoique nous ne le pensions pas " (8).

(3 bis) Il est juste de citer les analystes qui ont signé quelques comptes rendus: Beuchat, Bianconi, David, Davy, Doutté, Fauconnet, de Félice, Hertz, Lapie, Marx, Reynier, Roussel.

(4) Dans le no 20 des Archives, pp. 63-73, Joseph Sumpr a présenté «Durkheim ct le problème de l'étude sociologique de la religion ". Un article sera consacré à Marcel Mauss.

(5) Dans toutes les sections, la sociologie religieuse trouve sa place et son aliment. Une note complémentaire pourrait faire le bilan.

(6) Nous entendons : l'organisation constitutionnelle et administrative, les relations entre la société religieuse et la société profane, les échanges avec les puissances supranaturelles; tripartisme que nous avons appliqué au droit canon et justifié dans les Prolégomènes d'une Histoire du droit et des institutions de l'Eglise en Occident, où il est systématiquement adopté.

(7) A.S., II, pp. 1-28.

(8) A.S., II, préface IV. 
Mauss s'est expliqué sur le domaine propre à l'Année (9) et il a mis à part le culte des morts auxquels $\mathbf{R}$. Hertz a consacré un article, la mort étant « le passage de la société visible des vivants à la société invisible des ancêtres " (10).

Des religions, l'Année Sociologique s'applique à préciser les rapports avec les sectes. Une note brève et dense de Mauss reconnaît dans les sectes un phénomène d'une extrême généralité : il les signale dans toutes les religions, nationales ou universalistes, et affirme que "c'est sous la forme de sectes que naissent les religions " et, en somme, qu'elles manifestent une part de leur vitalité, les étapes d'un constant devenir (11).

Plus qu'à ces états des religions, Mauss s'est intéressé à ces activités en marge des religions que constitue la magie et "qui ne font pas partie d'un culte organisé, privé(s), secret(s), mystérieux et tendant comme limite vers le rite prohibé ॥. Avec la collaboration d'Hubert, il a identifié les éléments de la magie, qu'il analyse et explique : phénomène social qui s'apparente à la religion (le mana est du même ordre que la notion du sacré) et aux techniques (tendant au concret, alors que la religion tend à l'abstrait) (12).

La même curiosité des rapports entre magie et religion devait inspirer Huvelin (13). "Dans le domaine du droit, le rite magique n'est qu'un rite religieux détourné de son but social régulier et employé pour réaliser une volonté et une croyance individuelle. Illicite, secret, le rite magique « est religieux dans sa teneur extérieure, antireligieux dans ses fins" (14). Ses rapports avec la science sont reconnus (15).

Chez les peuples archaïques, le totémisme et les tabous sont une forme très répandue, probablement la plus courante des représentations et actions ayant quelque caractère religieux (15). La pensée de Durkheim n'a point la rigidité que lui prêtent trop de simplificateurs. D'abord "l'extension donnée à des termes spéciaux comme tabou, totem, déroute le savant et il en résulte souvent des erreurs. Le tabou n'est qu'un cas particulier d'un système plus général, celui des interdictions rituelles ; le totémisme, un cas des cultes thériomorphiques ou un cas des religions du clan. Il faut éviter de donner au genre le nom d'une espèce " (16). Mauss dénonce à nouveau les impropriétés du vocabulaire courant, rappelle que totem signifie culte thériomorphique de clan et préfère à tabou: interdiction rituelle (17).

Le genre n'est pas universel : il y a des sociétés archaïques sans totémisme et dont les croyances et les rites reposent sur des fondements plus larges que le totem (18). Enfin, le totémisme évolue (et conséquemment l'idée que s'en fait

(9) A.S., VII, pp. 199-201.

(10) "Contribution à une étude sur la représentation collective de la mort", A.S., X, pp. 48137. Voir également III, pp. 214-217, c. r. Rohde.

(11) A.S., VIII, pp. 293-95.

(12) "Esquisse d'une théorie générale de la magie ", A.S., VII, pp. 1-146.

(13) "Magie et droit individuel", A.S., X, pp. 1-47.

(14) A.S., I, p. 474: c.r. de Thorndike par H. Hubert.

(15) Durkheim, La prohibition de l'inceste et ses origines, I, pp. 1-70, notamment p. 39.

(16) A.S., II, p. 202.

(17) "Note sur la nomenclature des phénomènes religieux ", A.S., IX, pp. 248-251. Voyez déjà VIII, pp. 235-238 (Mauss, Le Totémisme).

(18) $A . S .$, III, p. 209. 


\section{ARCHIVES DE SOCIOLOGIE DES RELIGIONS}

Durkheim). Le premier ouvrage de Spencer et Gillea sur les Aruntas (19) en révèle une forme dénaturée, affaiblie, dont les phratries donnent la clé, qui se retrouvera utile pour comprendre les Urabunna (19 bis).

Contre toute contestation, le concept durkheimien est défendu avec vigueur (20).

De gros ouvrages concernant les religions de l'Antiquité ont suscité chez Henri Hubert des réflexions pénétrantes sur l'Egypte (21), la Grèce (22), et Rome (23), sur Israël et la Babylonie (24).

"Nous étudions peut-être trop «les primitifs» et pas assez les grandes religions, les nôtres, les mouvements de sentiments et d'idées qui les agitent", confesse Mauss (25). Il est vrai que le brahmanisme, l'islamisme, le bouddhisme (26) ni même le christianisme (27) ne tiennent pas dans l'Année Sociologique une place aussi originale que les "primitifs ». Cependant, les principaux ouvrages sur l'histoire générale des religions (28), l'histoire de chacune d'elles, les recherches de sciences religieuses (29) remplissent de longues pages d'Analyses. Les formes populaires excitent la curiosité (30).

Les religions archaïques sont encombrées de mythes et de rites dont certains se systématisent peu à peu en croyances et liturgies.

Mauss a longuement présenté et critiqué Wundt, pour qui le mythe domine le rite, qui n'en serait que la mise en acte : pas de rite sans mythe, tandis que le mythe peut n'imposer aucun ritualisme (31). Que le mythe primitif de l'âme ait

(19) E. Durkhem, "Sur le totémisme ", A.S., V, pp. 82-121 ; Hubert et Mauss, A.S., III, pp. 205-215 ; XII, pp. 101-104.

(19 bis) E. Durkheim, "Sur l'organisation matrimoniale des sociétés australiennes ", A.S., VIII, pp. 118-147; Hubert et MaUss, pp. 242-252.

(20) Contre Marillier : A.S., II, p. 204, V, pp. 191-197. Contre Frazer : A.S., II,p. 202, V, pp. 205-217, XII, pp. 75-79 et 91-95. Contre Reinach : A.S., IX, pp. 174-176.

(21) Sur Moret : A.S., VII, p. 289.

(22) Analyse de O. GruPPE, Griechische Mythologie und Religion, A.S., VI, pp. 237-242 et IX, pp. 325-328 (H.H. et M.M.) ; de HARISSON (H.H.), A.S., VIII, pp. 270-276 ; voyez encore III, pp. 310-314; VIII, pp. 296-299.

(23) Ainsi, l'analyse de A. de Marchi, Il culto privato di Roma antica, dans A.S., I, pp. 100197 : Mauss refuse de compter parmi ces cultes privés le culte des dieux de l'Etat. - Voyez encore IV, pp. 289-294; et S. Wissowa : VI, pp. 290-293 et VIII, pp. 278-281.

(24) Analyse dans A.S., IV, pp. 190-192, 201-203 ; III, pp. 235-241, 279-282, et 300-305.

(25) A.S., n. s., II, p. 123.

(26) Remarques sociologiques sur le brahmamisme : A.S., IV, pp. 52-59; X, p. 151 ; sur l'islam : la plus importante étude sur la religion populaire dans l'Islam est le mémoire de $\mathbf{R}$. Maunier, "Recherches sur les échanges rituels en Afrique du Nord ", A.S., n.s., II, pp. 11-97. La taoussa, échange rituel, usuraire et casuel, caché sous un don fictif,'commence par la prière, est un épisode des fêtes et procure la baraka. Sur le bouddhisme : A.S., IV, pp. 251-254. Les volumes de J.-J. de Groot sur les religions de la Chine ont été régulièrement analysés.

(27) Grand intérêt pour le christianisme primitif, A.S., VI, pp. 211-217, VIII, pp. 254-257;

IX, pp. 265-269. Mauss signale l'importance sociologique de Hauck, $A . S$., IV, pp. 209-212.

(28) Analyse des ouvrages de Byron Jevons, $A . S .$, I, p. 160; de Söderblom, A.S., V, pp. 235241 ; de Chantepie, VII, pp. 243-249.

(29) Psychologie religieuse: Analyse de Sabatier, de H. Koch, A.S., I, pp. 191-197; de Starbuck, $A . S$., IV, pp. 154-158. Voyez encore VII, pp. 201-204; VIII, pp. 227-234.

(30) A.S., I, pp. 210-218; II, pp. 197-201.

(81) A.S., XI, pp. 58-68. 
donné naissance à trois formes de culte (magie, fétichisme, totémisme) Mauss n'y semble point voir d'objection fondamentale, mais le passage du culte des héros éphémères aux dieux permanents lui paraît sans explication.

La difficulté préalable est de distinguer le mythe (proprement religieux) de la légende et du roman. Elle est aussi de suivre son développement jusqu'à un "système d'images et de notions qui les encadrent " (32): car les mythes comme les totems "ont des âges" (33).

La plupart des ouvrages sur le culte chrétien ont été l'objet d'analyses délicates et d'appels à développements sociologiques, dont l'importance était signalée dès le premier volume de l'Année Sociologique (34). Une attention particulière est accordée aux fêtes (35).

Le culte impose une discipline, qui règle les rapports à l'intérieur du groupe, et avec les puissances supranaturelles.

Il semble que l'organisation des sociétés religieuses, si importante pour le sociologue, a été abandonnée aux canonistes. La structure (c'est-à-dire la démographie et la morphologie, la coordination verticale, diagonale, horizontale), la vie (c'est-à-dire les accords et les tensions, les pratiques et les refus) tiennent peu de place dans les Mémoires et les analyses de l'Année Sociologique. Il faut cependant excepter le monachisme, dont les origines et les formes ont éveillé l'attention (36).

Le lien entre religion et société ne saurait être plus étroit que les rapports dans les clans : le totem nous l'a fait saisir. Il peut être aussi serré dans des sociétés parvenues à un plus grand degré de civilisation, comme le montre Bouglé au cours de ses "Remarques sur le régime des castes 》 (37). Aux Indes, l'influence des idées religieuses a été déterminante dans la hiérarchie des castes, et l'habitude des cultes fermés. Bien que le clergé y soit un corps, non une caste, le régime s'est maintenu plus longtemps que dans la plupart des sociétés. Chez les Eskimos, les variations de la société profane ont d'extraordinaires effets sur la vie religieuse (38).

Faire communiquer le monde sacré et le monde profane, c'est le but éminent des religions. Chez les peuples archaïques, le sacrifice a cet objet précis, comme le montrent Hubert et Mauss dans leur «Essai sur la nature et la fonction du sacrifice" (39).

(32) A.S., VI, p. 260.

(33) Ibid., p. 262. Sur les noms des dieux : A.S., I, pp. 240-247.

(34) Compte rendu de Mgr Fr. Magnani, L'Antica liturgia romana, vol. I, dans A.S., I, pp. 224-228 ; de CABrol, Les origines liturgiques, A.S., XI, pp. 212-213; de RiEtschel, A.S., IV, pp. 222-225.

(35) Par exemple, dans le vol. IV.

(36) Voyez notamment l'analyse des ouvrages de Zöckler dans $A . S .$, I, pp. 257-264, et II, pp. 274-277 de Hannay, A.S., VIII, pp. 365-369.

(87) $A . S .$, IV, pp. 1-64.

(38) Marcel Mauss, "Essai sur les variations saisonnières des societés Eskimos ", A.S., IX, pp. 39-130, spécialement, pp. 96-104 : pas de vie religieuse l'été, exaltation pendant l'hiver. (39) A.S., II, pp. 29-138. Il s'agit d'établir cette communication * par l'intermédiaire d'une victime, c'est-à-dire d'une chose détruite au cours de la cérémonie ". 


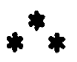

En 1924, l'Année annonçait la fondation d'un Institut français de Sociologie ; l'objet en était "de rapprocher les spécialistes des diverses sciences dont la réunion constitue la science de l'homme vivant en société " (40); composé de 50 membres titulaires, tous élus et qui, au bout de dix ans deviennent membres du Conseil.

Peu après ma nomination à Paris (1930), j'ai connu la plupart de ces sociologues et depuis 1935 j'ai été membre de leur Institut : qu'il soit permis à l'un des rares survivants (41) de porter témoignage.

Le public (où se rencontrent beaucoup d'universitaires) se représentait une secte, dans une chapelle, avec ses dogmes, ses clôtures et sa vanité. Que de fois ai-je recueilli cette légende, chez des maîtres savants et bons ! En fait, j'ai connu un groupe accueillant, sans préjugés, dont l'unique souci était de contribuer à l'avancement de la sociologie. Un "tala y respirait à l'aise aussi bien qu'un agnostique. Mauss, notre patriarche, savait tout, par charisme et répandait ses dons sans attendre le contre-don. La méditation continue, la modestie sans borne d'Halbwachs en faisaient comme un être surnaturel. La fine culture, l'exquise gentillesse de Bouglé, la puissante et austère pensée de Granet, le généreux prophétisme de Massignon nous séduisaient (42). Simiand savait la mathématique des dieux, Henri Lévy-Brühl leurs artifices juridiques et il leur dérobait des trésors de bonté.

L'Institut a publié un Bulletin qui n'eut pas longue vie (43) et des Annales sociologiques (1934-1939), qui ont paru en cinq séries avec le sous-titre : Collection de l'Année sociologique. Des séries A (Sociologie générale, 4 fascicules), C (Sociologie juridique et morale, 3 fascicules), D (Sociologie économique, 4 fascicules), rien à retenir pour notre enquête. Le fascicule 1-3 de la série B (Sociologie religieuse) est une étude en $\mathbf{2 5 0}$ pages de Marcel Granet sur les "Catégories matrimoniales et relations de proximité dans la Chine ancienne ".

La série $\mathrm{E}$ (Morphologie sociale, langage, technologie, esthétique) comprend 3 fascicules. Le second contient: Maurice Halbwachs, "La Morphologie religieuse" (p. 8-14) et Gabriel Le Bras, "Les Transformations religieuses des cam-

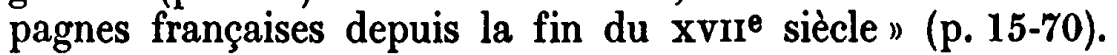

Telle m'apparait l'œuvre intellectuelle de ce groupe humain. Jusqu'en 1945, l'Année Sociologique a été la seule revue importante de sociologie publiée en français ; l'Institut, le seul organisme vivant. Il est donc juste de dire que nous avons évoqué en quelques pages un chapitre essentiel de l'histoire de la sociologie religieuse en notre pays. Mauss.

(40) L'ancien groupe de l'Année Sociologique se reconstituait sous l'autorité débonnaire de

(41) Des 48 membres de 1931, quatre seulement sont encore en vie : Marcel Cohen, G. Davy, R. Lenoir, Jean Marx.

(42) Curieux hasard : de lointaines parentés ou alliances m'unissaient à ces trois aînés : à Bouglé, par mon père ; à Granet par ma mère ; à Massignon par ma femme. Ces cousinages ne furent pour rien dans mon adhésion au groupe, mais ils m'en ont fait mieux sentir le caractère quasi-familial.

(43) J'en ai seulement le tome I (1930-1931), que m'a communiqué G. Davy. 
Nous n'avons pas écrit de l'Ecole, encore que les noms de Durkheim et de Mauss soient en tête du tableau. Ils étaient honorés de tous, mais une parfaite liberté de pensée permettait la diversité des opinions. Mieux qu'une Ecole, le groupe né dans le petit cercle de l'Année s'est épanoui dans toutes les Revues actuellement prospères : ni la Revue française de sociologie, ni les Cahiers internationaux de sociologie, ni les Archives de sociologie des religions ne contesteront leur dette envers les grands ancêtres.

Ceux d'entre nous qui se sont appliqués à la sociologie du catholicisme doivent à Durkheim une gratitude qui s'est exprimée le jour de la célébration du Centenaire de sa naissance (44). Dans un temps où la religion n'était pour la plupart des Français que sujet de passions aveugles et de bagarres parfois sanglantes, Durkheim a imposé l'étude sereine d'un fait social, et si toutes ses conclusions ne pouvaient, certes, rallier l'assentiment des chrétiens, elles étaient présentées avec calme et avec tact.

Me sera-t-il permis de reconnaître ma dette envers Marcel Mauss ? A partir de 1930, c'est-à-dire un peu avant mon premier article de sociologie religieuse, le voisinage a favorisé la rencontre de cet éveilleur remarquable et généreux. Quelques phrases de lui avaient déjà orienté ma réflexion. Dès 1907, analysant l'ouvrage d'Arréat, Le sentiment religieux en France, Mauss avait recommandé l'observation de toutes les manifestations de la vie religieuse et, avec force, les statistiques. En 1925, il précisait sa pensée en des termes qui nous ont beaucoup frappé. "La force d'une Eglise se mesure au nombre et à la richesse de ses temples, au nombre de ses croyants et à la grandeur de leurs sacrifices (...). Manié avec prudence et intelligence, le procédé statistique est non seulement le moyen de mesurer mais le moyen d'analyser tout fait social..." (45).

Dans un temps où tant d'hommes renient leur passé, bafouent leurs anciens amis, nous avons plaisir à écrire une modeste page d'histoire où la chronologie marque les étapes de notre gratitude.

Gabriel Le Bras. 1960.

(44) "Emile Durkheim sociologue de la religion ", dans Annales de l'L'niversité de Paris, (45) $A . S$. , nouv. série, II, p. 152. 